\title{
Morphological Variation in Selected Sri Lankan Rice (Oryza sativa L.) Accessions in Relation to the Vegetative Parameters
}

\author{
H.C.D. Wijayawardhana*, H.M.V.G. Herath ${ }^{1}$, P.A. Weerasinghe ${ }^{2}$ and \\ H.M.D.A.K. Herath ${ }^{2}$
}

\author{
Postgraduate Institute of Agriculture \\ University of Peradeniya \\ Sri Lanka
}

\begin{abstract}
Morphological variation of 16 rice accessions representing both traditional and improved varieties was assessed using 12 vegetative traits as described in International Rice Research Institute descriptor for rice, in sand medium using a Complete Randomized Design. The single linkage clustering, morphological dendrogram, multivariate analysis of variance and the Principal Components Analysis were performed to assess the traits. According to the cluster analysis, accessions 2840 and 10617 were the most closely associated among the varieties. Based on the variations associated with morphologically important vegetative traits, all the rice accessions were classified into three major groups as two traditional and one new improved variety groups. Existence of such clusters was validated by the Wilks' lambda statistics. The first four principle components explained over $80 \%$ of the total variation associated with the varieties. Among them the first two principle component cumulatively explained $54.46 \%$ of the total variation. Rice accessions 8920 and 2835 representing Bg360 and Bg 450, respectively showed the highest principle component 1 scores. Traditional rice varieties showed the comparatively higher scores in both principle components 1 and 2 than newly improved varieties. This study indicated that measured vegetative agro-morphological traits were helpful for preliminary characterization of varieties and also they can be used as a broad-spectrum approach to assess morphological diversity among morphologically distinguishable rice accessions.
\end{abstract}

Keywords: Morphological variation of rice, multivariate analysis, new improved varieties, traditional varieties

\section{INTRODUCTION}

Rice (Oryza sativa L.) is considered as one of the most important cereal crops and the staple food for more than half of the world's population (Jiang et al., 2013). It is considered as the ideal model plant to study grass genetics and genome organization due to its diploid nature, comparatively small genome size (Causse et al., 1994; Kurata et al., 1994), considerable level of genetic polymorphism (McCouch et al., 1998), a large amount of well conserved genetically diverse materials and fully sequenced genome (Pervaiz et al., 2010; Rabbani et al., 2010). Although the world rice production has doubled in past 30 years due to the introduction of superior varieties and better cultivation practices as a result of green revolution, it is still insufficient to reach the increasing global demands (Fischer et al., 2000;

Department of Agricultural Biology, Faculty of Agriculture, University of Peradeniya, Sri Lanka

2 Department of Plant Sciences, Faculty of Agriculture, Rajarata University of Sri Lanka, Anuradhapura, Sri Lanka

Corresponding author: dwdarshani@yahoo.com 
Sasaki \& Burr, 2000). It is estimated that the total demand for rice in the world would increase at about $1 \%$ per annum from 2001 to 2025(Maclean et al., 2002).

Sri Lankan rice germplasm exhibits a wide diversity ranging from lowland to upland conditions. Currently most of the rice lands in the country are cultivated with NIVs and occasionally some traditional and old improved varieties are cultivated in minor extents (Rajapakse et al., 2000). Although Newly Improved Varieties (NIVs) produce comparatively higher yields, preference is higher for traditional rice varieties due to their high nutritional value, medicinal properties and the ability to withstand extreme weather conditions, diseases and pests. In addition, farmer's preference (Efisue et al., 2008) and the grain qualities such as high fiber content (Wickramasinghe \& Noda, 2008) also make interest towards the cultivation and utilization of traditional rice varieties. International Rice Research Institute (IRRI), Philippines identified Sri Lanka as one of the important geographical origins of many useful rice traits. The major traits are broad based resistance to East Asian biotypes of brown plant hopper, green leaf hopper, white-backed plant hopper, rice thrips and tolerance to salinity, iron toxicity, drought and other adverse soil conditions (Arachchi \& Wijerathna, 2008).

Due to the importance of rice as one of the major world food crops, its genetic diversity has attracted great interest of researchers. The genetic diversity of rice has been used effectively to increase the productivity. The majority of programs involved in improvement of rice productivity has been mainly focused on the yield aspects meanwhile other important agromorphological characters have been neglected. It is essential that not only to conserve the existing genotypes but also to explore the gene-pool characteristics to capture such important agro-morphological characters. In favor of this reason, it is crucial to discover the genetic diversity and the relationships among cultivars. Morphological evaluation is a preliminary step to estimate the variability and relationship among cultivars although several other tools are also used extensively (Smith et al., 1991).Therefore, to establish a country specific successful crop improvement programme, it is very much essential to discover the morphological variability of existing rice cultivars within the country. Even though Sri Lanka has nearly 68 released rice varieties by the Department of Agriculture and 2000 indigenous rice varieties, the total diversity of the indigenous varieties has not been exploited in more detailed manner. This study was designed to evaluate the morphological diversity that exists among selected Sri Lankan rice varieties using parameters of the vegetative growth stage with the objectives of uncovering the diversity of local germplasm and to develop a standard evaluation method that can be effectively used for local crop improvement programs.

\section{METHODOLOGY}

\section{Planting materials and experimental design}

Seeds of 16rice accessions representing both improved (10 accessions) and traditional (6 accessions) varieties as in Table 1, were selected by considering the popularity among Sri Lankan farmers and particular seeds were obtained from Plant Genetic Resources Center (PGRC), Gannoruwa, Peradeniya, Sri Lanka. Seeds were germinated on petridishes and after 10 days, two uniform healthy seedlings were transplanted in each pot containing $10 \mathrm{~kg}$ of sand as potting medium. Experiment was carried out in the plant house of Faculty of Agriculture, Rajarata University of Sri Lanka and pots were arranged in complete randomized design with three replicates. Urea, Triple super phosphate and Murate of potash 
were used to provide major nutrients as recommended by the Department of Agriculture, Sri Lanka (Fertilizer usage in paddy cultivation, 2010) and Yoshida nutrient media (Yoshida et al.,1976) was used to provide micronutrients.

Table 1. Different rice accessions used for the study

\begin{tabular}{lccc}
\hline Serial No. & $\begin{array}{c}\text { Variety } \\
\text { group }\end{array}$ & $\begin{array}{c}\text { Accession } \\
\text { No. }\end{array}$ & $\begin{array}{c}\text { Name of the } \\
\text { variety }\end{array}$ \\
\hline 1 & & 2851 & $\mathrm{H} 4$ \\
2 & & 2840 & $\mathrm{Bg} 300$ \\
3 & 10617 & $\mathrm{Bg} 305$ \\
4 & & $\mathrm{Bg} 350$ \\
5 & Improved & 7182 & $\mathrm{Bg} 352$ \\
6 & varieties & 8919 & $\mathrm{Bg} 357$ \\
7 & & 8923 & $\mathrm{Bg} 359$ \\
8 & 8920 & $\mathrm{Bg} 360$ \\
9 & & 2835 & $\mathrm{Bg} 450$ \\
10 & & 9202 & Ld356 \\
\hline 11 & & 4740 & Kaluheenati \\
12 & & 2340 & Wedaheenati \\
13 & & 3136 & Pachchaperumal \\
14 & & 3809 & Murungakayan \\
15 & Traditional & 3223 & Rathkanda \\
16 & varieties & 5566 & Raththawalu \\
\hline
\end{tabular}

\section{Data collection}

Evaluation of the rice accessions was carried out for different morphological parameters representing the vegetative growth stage of rice. Trait selection and measurement techniques were based on IRRI standard evaluation system of rice (IRRI, 1980). Leaf length (length of the topmost leaf blade below the flag leaf on the main culm in centimeters), leaf width (width of the widest portion of the blade on the leaf below the flag leaf in centimeters), leaf angle (the angle of the openness of the blade tip against the culm on the leaf below the flag leaf), ligule length (length from the base of the collar to the tip of the ligule in millimeters), culm length (length from the ground level to the base of the panicle in centimeters), culm number (total number of tillers)were measured accordingly and the other parameters such as leaf blade pubescence, leaf blade color, basal leaf sheath color, ligule color, internode color, auricle color, ligule shape and culm angle were measured using IRRI descriptors for (Oryza sativa L.) and photographs.

\section{Data analysis}

All recorded agro-morphological traits were analyzed using two complementary procedures; Cluster and Principal Component Analysis (PCA).Single linkage cluster analysis was performed to group the rice accessions and Principle Components PCs) analysis was performed to assess the pattern of variation among the parameters with the same data matrix (Ogunbaya et al., 2005). Score plots of first three PCs were used to provide a graphical representation of the pattern of variation among the rice accessions. Multivariate analysis of variance (MANOVA) procedure was used to assess the significance of the performed 
clusters. These tests were performed with the Statistical Analysis System software (SAS, Version 9.0).

\section{RESULTS AND DISCUSSION}

\section{Variability of measured parameters}

The evaluation of morphological traits usually reveals important traits which are essential to characterize the genetic resources. Out of the total evaluated traits, two agro-morphological characters; ligule shape and auricle color were omitted from the analysis due to lack of variability among the tested rice accessions. The other tested traits showed a considerable level of variability among the tested rice accessions. Therefore, these traits were taken for further analysis. Figure 1 shows the variation in internode color observed in the accessions.

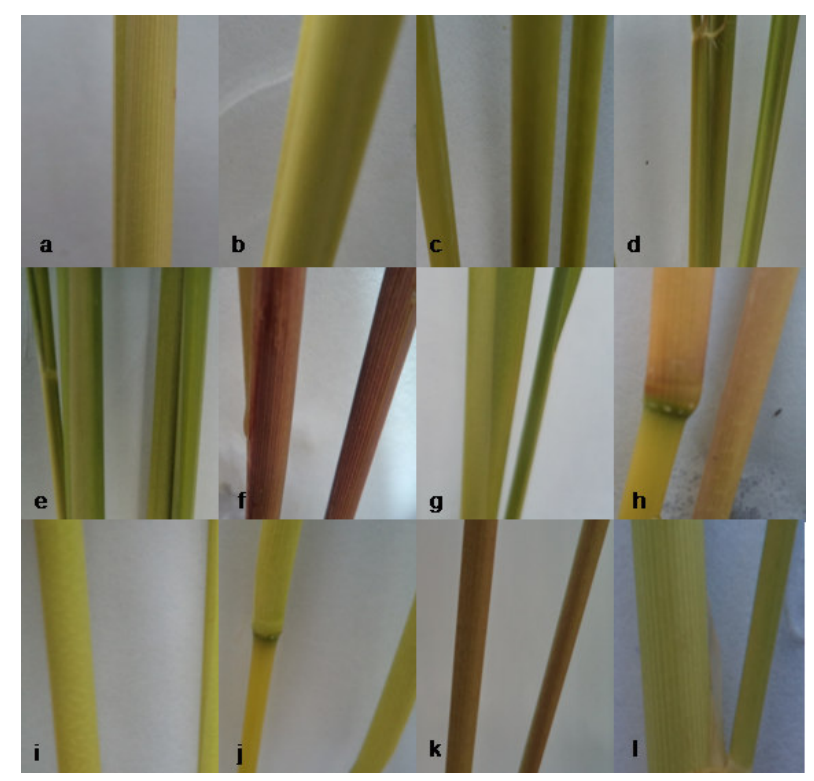

Fig. 1. Variation in internode color of rice accessions. (a-2840:Bg300, b10617:Bg305, c-2837:Bg350, d-7182:Bg352, e-8919:Bg357, f-8920:Bg360, g-9202:Ld356, h-4740:Kaluheenati, i-2340:Wedaheenati, j-3136:Pachchaperumal, k-3223:Rathkanda, l-5566:Raththawalu)

\section{Cluster analysis}

All rice accessions were grouped into 3 clusters at 0.5 minimum distance between clusters (Fig. 2). Numbers of rice accessions in these 3 clusters were 4, 2 and10in the first, second and the third clusters, respectively. When consider the grouping behavior of rice accessions, a clear differentiation of rice accessions into three clusters was observed with few exceptions. Thus, cluster I and II represented the rice accessions for traditional varieties and cluster III represented the rice accessions of NIVs. Interestingly, accession 2851 reflecting old improved variety $\mathrm{H} 4$ grouped into the cluster I containing accessions for traditional varieties. Furthermore, accession 3136 represent the traditional variety Pachchaperumal clustered into the third cluster which contained all the NIVs rice accessions. Rice accessions 
2840 and 10617 representing NIVs, Bg300 and Bg305 respectively were first formed in to a group at 0.12 minimum distance between clusters. Therefore, these two accessions can be considered as morphologically closest accessions among the tested ones.

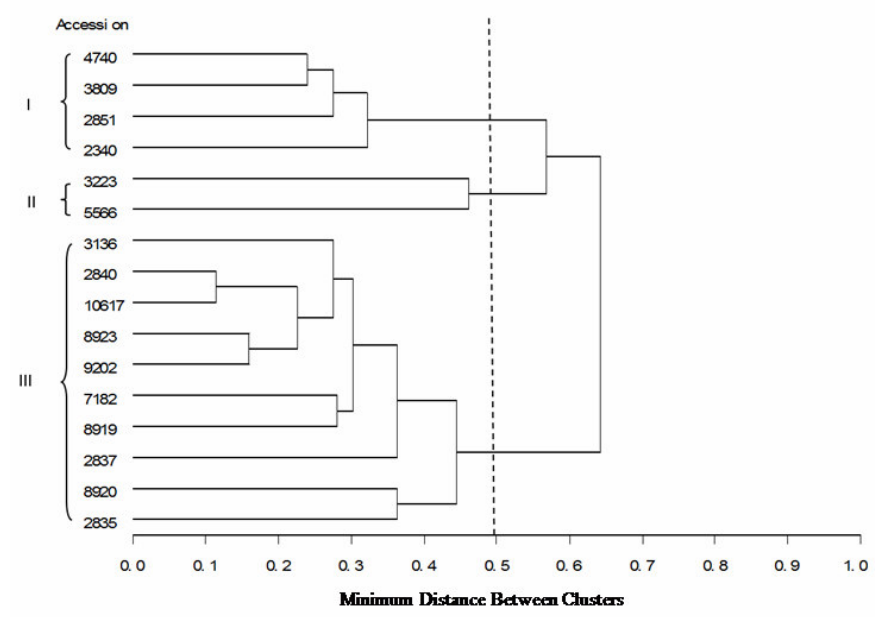

Fig. 2. Dendrogram of rice accessions obtained through single linkage cluster analysis. Dashed line indicated the minimum distance between clusters. The clusters were identified as I, II and III

\section{MANOVA analysis}

The MANOVA analysis of the above 3 clusters against the 12 measured variables revealed a significant Wilks' Lambda statistics $(\mathrm{p}<0.0001)$. Therefore, these three rice groups classified according to the resulted data are truly different from each other and they are naturally existing clusters. Thus tested vegetative traits can be utilized effectively to group the Sri Lankan rice accessions.

\section{Principle component analysis}

Principle component analysis showed that the first four PCs having eigen values greater than 1 accounted for $84.78 \%$ of the total variation. The first principle component individually explained nearly one third of the total variation whereas the first two principle components cumulatively explained the $54.46 \%$ of the total variation and the combination of principle component 1 (PC1) and principle component 3 (PC3) explained the $45.96 \%$ of the total variation covered by all rice accessions (Table 2).Among NIVs, rice accessions of 8920 (Bg360) and 2835 (Bg450)showed extremely high positive PC1 scores, reflecting highest contribution from internode color, ligule color, basal leaf sheath color and leaf blade pubescence, while accessions of 10617 (Bg305), 9202 (Ld356), 8923 (Bg359), 7182 (Bg352), 2840 (Bg300), 8919 (Bg357) and 2837 (Bg350) showed the comparatively low PC1 scores (Fig. 3) reflecting lowest culm length and leaf width (Table 2). 
Table 2. Variation among rice accessions accounted for first four principle components

\begin{tabular}{lcccc}
\hline Parameter & PC1 & PC2 & PC3 & PC4 \\
\hline Leaf length & 0.346 & 0.384 & 0.120 & 0.054 \\
Leaf width & -0.157 & -0.377 & -0.412 & 0.044 \\
Leaf blade pubescence & 0.349 & 0.099 & -0.258 & -0.293 \\
Leaf blade color & 0.258 & -0.348 & 0.062 & 0.402 \\
Basal leaf sheath color & 0.373 & -0.110 & 0.357 & -0.115 \\
Leaf angle & 0.016 & -0.087 & 0.529 & 0.418 \\
Ligule length & 0.209 & 0.441 & -0.305 & 0.062 \\
Ligule color & 0.417 & -0.258 & -0.149 & -0.130 \\
Culm length & -0.008 & 0.481 & -0.105 & 0.320 \\
Culm number & 0.072 & 0.101 & 0.427 & -0.541 \\
Culm angle & 0.349 & 0.043 & -0.035 & 0.372 \\
Internode color & 0.425 & -0.229 & -0.160 & -0.054 \\
Eigen value & 3.631 & 2.903 & 1.882 & 1.755 \\
Proportion of the variance \% & 30.27 & 24.19 & 15.69 & 14.63 \\
Cumulative variance \% & 30.27 & 54.46 & 70.15 & 84.78 \\
\hline
\end{tabular}

Principle component 2 (PC2) obtained $24.19 \%$ of the total morphological variability. Rice accessions of traditional varieties showed the highest positive PC2 scores compared with accessions for NIVs, except for the accession of 3136 (Pachchaperumal), reflecting the higher culm length, ligule length and leaf length (Table 2). Figure 3 shows the plot obtained from the first two eigenvectors of the PCs analysis.

The PC3 accounted for $15.69 \%$ of the total variation. Rice accession 3136 (Pachchaperumal)had the comparatively highest PC3 score than the other all accessions of the traditional varieties, indicating highest leaf angle and culm number (Table 2). Figure 4 shows the plot obtained from the first and third eigenvectors of the PC analysis. Furthermore, rice accessions for NIVs showed comparatively low scores for both PC1 and PC2 than the accessions for traditional varieties except for the accessions 8920 (Bg360) and 2835 (Bg450). These results are possible due to the morphological changes achieved through the successive rice improvement programs. Along with the PC3 such distinguishable differentiation in between traditional and NIVs groups could not be observed.

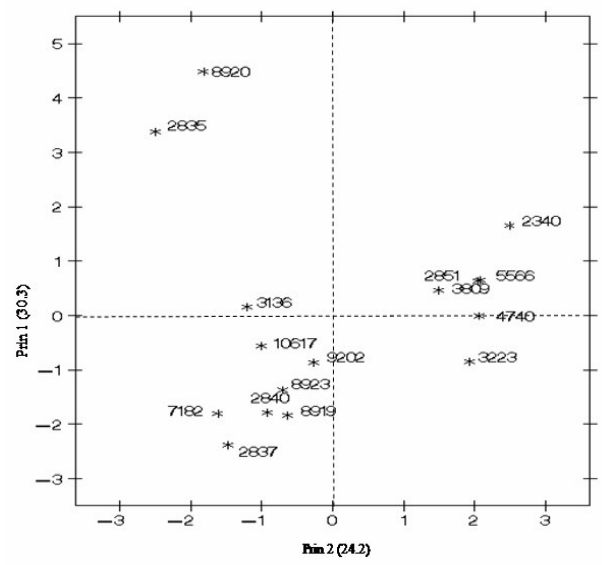

Fig. 3. The score plot of PC1 (Prin 1) and PC2 (Prin 2) based on vegetative characters of accessions 
Results of the present study revealed that the all tested rice accessions harbor a considerable range of morphological variation. According to Ali et al. (2000), cluster analysis has the singular efficacy and ability to identify crop accessions with highest level of similarity. The dendrogram obtained from the present study also proved the above statement in terms of similarity existing among accessions. Even though rice varieties are broadly classified in to two groups as traditional and NIVs, a wide variability among them was identified. Based on the grouping behavior of the tested rice accessions, such variability could be observed. Further, Tehrim et al. (2012) also proved that agro-morphological traits can be used effectively to characterize the rice cultivars.

Suriyagoda et al. (2011) have reported a similar variability of rice varieties. Results of the present study have shown similarities to the findings of Suriyagoda et al. (2011) in terms of dendrogram analysis, clustering groups and PCs analysis with some exceptions. These exceptions were identified in terms of variations in cluster formation and grouping behaviors of their tested rice varieties. Further, these exceptions are possible due to the variations in external conditions such as soil types, and soil fertility levels (Steel, 1972) and soil moisture regimes (Morakinyo and Ajibade, 1998) associated with two cropping systems. Furthermore, the genetic make-up of seed, environment and field management practices have been reported to influence the morphology of a crop (Singh and Rachie, 1985).

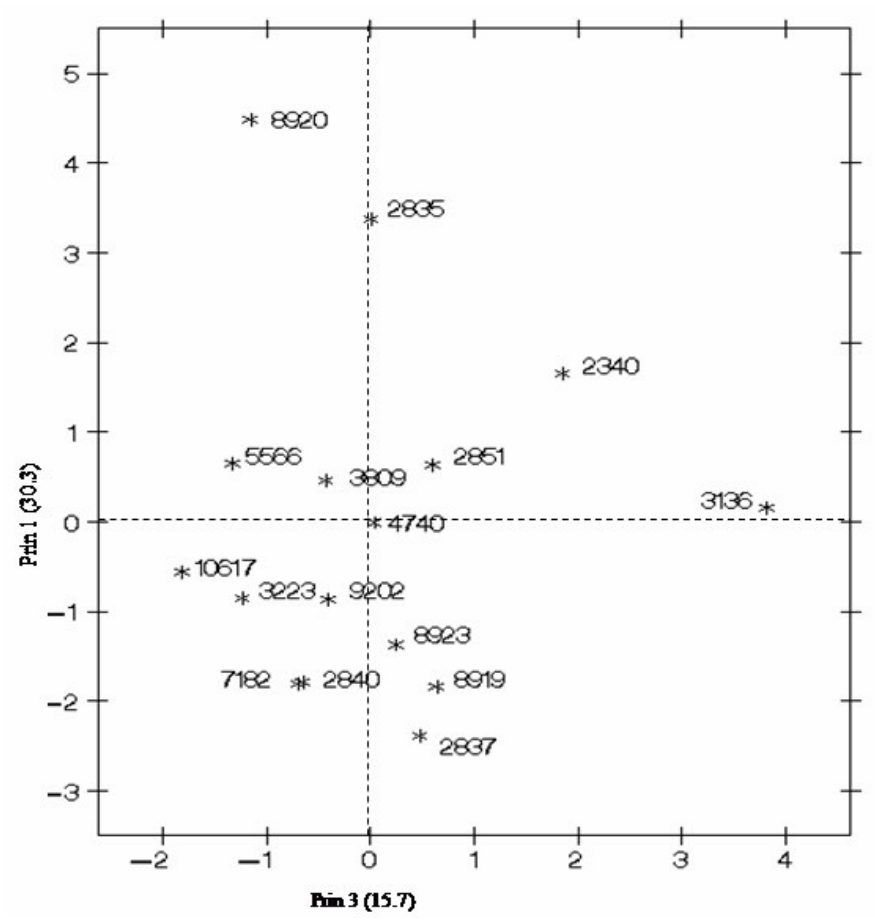

Fig. 4. The score plot of PC1 (Prin 1) versus PC3 (Prin 3) based on vegetative characters of accessions

Results of the present experiment have clearly indicated the importance of use of measured agro-morphological traits to identify naturally existing distinguishable clusters. With the use 
of this information, plant breeders can effectively select morphologically more distinct individuals for their breeding programs. Not only that, to meet the future rice demand in Sri Lanka with the increasing population, one option is to increase the productivity per unit area of the land, thus the identification of more yield related agro-morphological characters is very much important. Furthermore, the Department of Agriculture, Sri Lanka has already identified that the development of inbred rice varieties with a new plant architecture as one of the avenues in future rice improvement programmes (Rajapakse et al., 2012). Therefore, the identification of agro-morphological characters such as number of tillers that are important to change the rice crop architecture has a greater implication in this attempt. Hence, the data from the current study with other agro-morphological data may be widely applicable in future rice crop improvement programmes.

\section{CONCLUSIONS}

Morphological traits are used as a preliminary evaluation tool due to their easiness and can be employed as a common approach for assessing genetic variability among phenotypically distinguishable rice accessions. This study was highly focused on the vegetative characters of the rice plant irrespective of the reproductive characters. Most of the previous studies have reported the morphological variability in favor of both vegetative and reproductive characters. However, the present study, interprets a considerable amount of morphological diversity along with the vegetative traits of the rice plant. Therefore, rice vegetative traits can be used effectively in order to capture a considerable morphological variability associated with rice germplasm.

\section{ACKNOWLEDGEMENT}

The authors gratefully acknowledge the financial support from Rajarata University research grant RJT/ RP\&HDC/2013/Agric./R/04 for the financial support for this work and for PGRC-Gannoruwa for providing the seed materials of all tested rice accessions. We also acknowledge the Dr. W.C.P. Egodawatta and Mr. A.M.K.R.Bandara for providing assistance to statistical analysis.

\section{REFERENCES}

Ali, S.S., Jafri, S.J.H., Khan, T.Z., Mahmood, A. and Butt, M.A. (2000). Heritability of yield and yield components of rice.Pak. J. Agric. Res. 16, 89 - 91.

Arachchi, D.H.M. and Wijerathne, P.M. (2008). The state of diversity.Pp. 11. In: Country report on the state of plant genetic resources for food and Agriculture, Sri Lanka. Department of Agriculture.

Causse, M.A., Fulton,T.M., Cho, Y.G., Ahn, S.N., Chunwongse, J. and Ronald, P.C. (1994). Saturated molecular map of the rice genome based on an interspecific backcross population. Genetics. 138, 1251 - 1274.

Efisue, A., Tongoona, P., Derera, J., Langyintuo, A., Laing, M., Ubi, B. (2008). Farmers' perceptions on rice varieties in Sikasso region of Mali and their implications for rice breeding.Journal of Agronomy and Crop Sci. 194, 393 - 400. 
Fertilizer usage in paddy cultivation (2010). Extension and Training center, Department of Agriculture, Peradeniya.

Fischer, K.S., Barton, J., Khush, G.S., Leung, H. and Cantrell, R. (2000). Collaborations in Rice Sci. 290, 279 - 280.

International Rice Research Institute and International Board for Plant Genetic Resources (1980). Descriptors for rice Oryza sativa L.The international Rice Research Institute, Manila, Philippines.

Jiang, S.H., Zhou, H., Lin, D.Z., Dong, Y.J., Ye, S.H. and Zhang, X.M. (2013). Identification and gene mapping of a thermo-sensitive leaf-color mutant at seedling stage in rice. Chin $\mathrm{J}$ Rice Sci. 27, 359 - 364.

Kurata, N., Nagamura, Y., Yamamot, K., Harushima, Y., Sue, N., Wu, J., Antonio, B.A., and Shomura, A. (1994). A 300 kilo base interval genetic map of rice including 883 expressed sequences. Nature Genet. 8, 365 - 372.

Maclean, J.L., Dawe, D.C., Hardy, B. and Hettel, G.P. (2002). Rice almanac. Los Banos (Philippines), International Rice Research Institute, Bouaké (Côte d'Ivoire), West Africa Rice Development Association, Cali (Colombia), International Center for Tropical Agriculture, Rome (Italy). Food and Agriculture Organization. 253.

McCouch, S.R., Kochert, G., Yu, Z.H., Wang, Z.Y. and Khush, G.S. (1998). Molecular mapping of rice chromosomes. Theor. Appl. Genet. 76, 815 - 829.

Morakinyo, J.A. and Ajibade, S.R. (1998). Characterization of the segregants of an improved cowpea line IT84K-124-6. Nig. J. Sci. 32, 27 - 32.

Ogunbayo, S.A., Ojo, D.K., Guei, R.G., Oyelakin, O.O. and Sanni, K.A. (2005). Phylogenetic diversity and relationships among 40 rice accessions using morphological and RAPDs techniques. African J. of Biotech. 4, 1234 - 1244.

Pervaiz, Z.H., Rabbani, M.A., Khaliq, I., Pearce, S.R. and Malik, S.A. (2010). Genetic diversity associated with agronomic traits using microsatellite markers in Pakistani rice landraces. Elect. J. Biotech. 13, 1 - 12.

Rajapakse, R.M.T., Sandanayake, C.A. and Pathinayake, B.D. (2000). Foot prints in rice variety improvement and its impact on rice production in Sri Lanka. Annual symposium of the Department of Agriculture, Sri Lanka, 2, 423 - 433.

Rabbani, M.A., Masood, M.S., Shinwari, Z.K. and Yamaguchi-Shinozaki, K. (2010).Genetic analysis of basmati and non-basmati Pakistani rice (Oryza sativa L.) cultivars using microsatellite markers.Pak. J. Bot. 42, 2551 - 2564.

SAS Institute Inc. (1999). SAS/STAT User's guide.Version 8.SAS Institute, Cary, North Carolina, USA.

Sasaki, T. and Burr, B. (2000). International rice genome sequencing project: the effort to completely sequence the rice genome. Curr.Opin. Plant Biol. 3, 138 - 141. 
Singh, S.R. and Rachie, K.O. (1985).Cowpea Research, Production and Utilization. John Wiley and Sons, New York.

Smith, S.E., Doss, A. and Warburton, M. (1991). Morphological and agronomic variation in North African and Arabian alfalfas.Crop Sci. 31, 1159 - 1163.

Steel, W.M. (1972). Cowpeas in Nigeria. Ph.D Thesis. University of Reading.

Suriyagoda, L.D.B., Thilakarathne, R.M.M.S., Nissanka, S.P. and Samitha, S. (2011). Morphological variation in selected rice (Oryza sativa L.) germplasm of Sri Lanka. J. Natn. Sci. Foundation Sri Lanka. 39, 129 - 137.

Tehrim, S., Pervaiz, Z.H., Mirza, M. Y., Rabbani, M. A., and Masood, M.S. (2012).Assessment of phenotypic variability in rice (Oryza sativa L.) cultivars using multivariate analysis. Pak. J. Bot. 44, 999 - 1006.

Wickramasinghe, H.A.M. and Noda, T. (2008). Physicochemical properties of starches from Sri Lankan rice varieties. Food Science and Technology Research. 14, 49 - 54.

Yoshida, S.I., Forno, D.A., Cock, J.H., Gomez, K.A. (1976). Laboratory manual for physiological studies of rice. International Rice Research Institute, Manila, Philippines. 